\title{
Evaporação Cutânea e Respiratória em Ovinos sob Altas Temperaturas Ambientes
}

\author{
Roberto Gomes da Silva1, Josiane M. Cardoso Starling ${ }^{2}$
}

RESUMO - Dez ovinos da raça Corriedale foram avaliados para as taxas de evaporação respiratória $\left(\mathbf{E}_{\mathrm{R}}\right)$ e cutânea $\left(\mathbf{E}_{\mathrm{C}}\right)$. Cada animal foi observado até 10 vezes, sob diferentes condições de temperatura $\left(21,1\right.$ a $\left.41,9^{\circ} \mathrm{C}\right)$ e pressão parcial de vapor do ar $(1,53$ a $3,01 \mathrm{kPa})$, usando um método gravimétrico para determinar a perda de água. As médias totais foram $0,7599 \pm 0,0094 \mathrm{~g} \cdot \mathrm{h}^{-1} \cdot \mathrm{kg}^{-1} \mathrm{para} \mathbf{E}_{\mathrm{R}} \mathrm{e}$ $1,3029 \pm 0,0591 \mathrm{~g} \cdot \mathrm{h}^{-1} \cdot \mathrm{kg}^{-1}$ para $\mathbf{E}_{\mathrm{C}}$. A evaporação cutânea foi considerada como um fator importante de termólise para ovinos em ambientes quentes. São discutidos os efeitos do sexo, da espessura do velo e da temperatura e umidade do ar.

Palavras-chave: evaporação respiratória, evaporação cutânea, ovinos, termorregulação

\section{Cutaneous and Respiratory Evaporation Rates of Sheep in Hot Environments}

\begin{abstract}
Ten adult Corriedale sheep were evaluated for respiratory $\left(\mathbf{E}_{\mathrm{R}}\right)$ and cutaneous $\left(\mathbf{E}_{\mathrm{C}}\right)$ evaporation rates. The animals were observed about 10 times each by recording changes in their live weigth with high-sensitiviy strain gauges, under different conditions of air temperature $\left(21.1\right.$ to $\left.41.9^{\circ} \mathrm{C}\right)$ and partial vapour pressure $(1.53$ to $3.01 \mathrm{kPa})$. Average evaporation rates were $0.7599 \pm 0.0094 \mathrm{~g} . \mathrm{h}^{-1} . \mathrm{kg}^{-1}$ for $\mathbf{E}_{\mathrm{R}}$ and 1.3029 $\pm 0.0591 \mathrm{~g} \cdot \mathrm{h}^{-1} \cdot \mathrm{kg}^{-1}$ for $\mathbf{E}_{\mathrm{C}}$. Cutaneous evaporation was considered as an important heat loss mechanism for sheep in hot environments. There were discussed the effects of sex, fleece thickness, air temperature, and air humidity.
\end{abstract}

Key Words: cutaneous evaporation, heat loss, respiratory evaporation, sheep

\section{Introdução}

Nos ambientes tropicais, a temperatura do ar tende a ser próxima da ou maior que a corporal, o que torna ineficazes os mecanismos de transferência térmica por condução e convecção. Em adição, a carga térmica radiante é freqüentemente muito elevada, de modo que a superfície corporal dos animais tende a apresentar temperaturas bastante altas, dependendo da natureza dessa superfície. No caso de ovinos expostos ao sol, têm sido observadas temperaturas de até $90^{\circ} \mathrm{C}$. Em conseqüência, a evaporação torna-se o mecanismo de termólise mais eficaz nesses ambientes, por não depender do diferencial de temperatura entre o organismo e a atmosfera. De fato, as perdas térmicas por evaporação em ovinos sob estresse de calor podem atingir $90 \mathrm{~W} . \mathrm{m}^{-2}$ (Monteith \& Unsworth 1990).

As evidências encontradas na literatura acerca da importância relativa da evaporação respiratória e da cutânea nos ovinos são conflitantes. Alguns autores mais antigos (Rieck 1950; Knapp \& Robinson, 1954; Brook \& Short, 1960 a,b) afirmam que, sob altas temperaturas $\left(30 \mathrm{a} 40^{\circ} \mathrm{C}\right)$, a evaporação cutânea chega a ser até três vezes mais intensa que a respiratória. Outros supõem que a evaporação respiratória seja mais importante (Alexander \& Williams, 1962; Hofmeyr et al., 1969; Hales, 1974).

É sabido que os ovinos possuem glândulas sudoríparas do tipo apócrino, associadas aos folículos pilosos primários de lã (Bligh, 1961; Robertshaw, 1966) e alguns autores, como Brook \& Short (1960a,b) e Alexander \& Williams (1962), determinaram altas taxas de sudação em animais tosquiados mantidos a $40^{\circ} \mathrm{C}$. Silva et al. (1990) observaram animais da raça Ideal sob temperaturas entre 25 e $46^{\circ} \mathrm{C}$, determinando uma taxa de sudação média de $90,5 \pm 5,5 \mathrm{~g} \cdot \mathrm{m}^{-2} \mathrm{~h}^{-1}$. Por outro lado, foi sugerido por Brockway et al. (1965) que, em animais cobertos de velo espesso submetidos a altas temperaturas em calorímetros, as perdas térmicas por evaporação respiratória seriam mais importantes que as cutâneas, alcançando $0,84 \mathrm{~W} \cdot \mathrm{kg}^{-1}$ a $30^{\circ} \mathrm{C}$. Hofmeyr et al. (1969) observaram que em ovinos não tosquiados expostos por duas horas a $38^{\circ} \mathrm{C}$ a evaporação respiratória constituía $65 \%$ da perda evaporativa total.

Deve ser ainda notada a variedade de unidades de medida adotadas pelos diferentes autores, alguns

\footnotetext{
${ }^{1}$ Professor Titular, Departamento de Zootecnia, Faculdade de Ciências Agrárias e Veterinárias, UNESP. 14884-900 Jaboticabal, SP. Email: rgomes@fcav.unesp.br

${ }^{2}$ Professora Assistente Doutora, Faculdade de Veterinária, Universidade de Franca, Franca, SP. E.mail: barbante@fcav.unesp.br
} 
se referindo à área de superfície corporal e outros ao peso corporal. Essas diferenças dificultam bastante a comparação dos dados da literatura.

O propósito do presente trabalho é investigar a importância relativa da evaporação cutânea e da evaporação respiratória para ovinos de uma raça originária de climas temperado e criados em uma região intertropical.

\section{Material e Métodos}

Foram utilizados dez animais adultos da raça Corriedale, sendo sete machos e três fêmeas, pesando entre 59,8 e $97,1 \mathrm{~kg}$, os quais não foram tosquiados antes nem durante as observações.

Para a determinação da evaporação total, foi empregado o sistema de pesagem de precisão WM204 (Scale-Tronix, Inc., White Plains, NY, USA), associado a uma gaiola metálica para contenção do animal sob medição. Este sistema consiste basicamente em quatro transdutores de pressão do tipo strain gauge, colocados sob um suporte onde se achava a gaiola, sendo conectados a uma unidade central computadorizada que registra continuamente as alterações no peso do indivíduo, com uma sensibilidade de $10 \mathrm{~g}$. A capacidade do sistema atinge cerca de $500 \mathrm{~kg}$. Esse equipamento é o mesmo empregado por outros pesquisadores (Holmes, 1985; Finch, 1985) para a determinação da taxa total de evaporação em bovinos.

O piso da gaiola era composto por uma placa de aço perfurada, através da qual as fezes e a urina podiam passar livremente, sendo colhidas em uma bandeja com óleo colocada embaixo. A função do óleo era impedir a evaporação da água dos dejetos durante a operação, evitando alterações indevidas no peso. O sistema completo era instalado no interior de uma câmara climática, na qual a temperatura variaou entre 21,1 e $41,9^{\circ} \mathrm{C}$ e a pressão parcial de vapor entre 1,53 e $3,01 \mathrm{kPa}$, conforme o dia.

Em cada sessão de observação, um animal era colocado na gaiola durante duas horas, sendo seu peso monitorado continuamente pelo dispositivo; ao término desse tempo, anotava-se a perda total de peso. Antes e depois desse processo eram tomadas a temperatura retal e a frequiência respiratória, anotando-se as respectivas médias. Foram feitas até 10 observações sucessivas em cada animal, num total de 81 observações nos meses de Setembro e Outubro, com um intervalo mínimo de uma semana entre as determinações em um mesmo animal.
Para determinação da evaporação respiratória, foi utilizado o método desenvolvido por Silva et al. (2002):

$$
\mathbf{E}_{\mathrm{R}}=60 \mathrm{v} F \Psi W^{-1}
$$

$\left(\mathrm{g} \cdot \mathrm{h}^{-1} \cdot \mathrm{kg}^{-1}\right)$, em que $\mathrm{v}=0,0496 \mathrm{~F}-1,1557$ é o volume respiratório corrente $\left(\mathrm{m}^{3}\right.$ de ar por respiração); $\mathrm{F}$, a frequiência respiratória $\left(\mathrm{min}^{-1}\right) ; \mathrm{W}$, o peso corporal $(\mathrm{kg}) \mathrm{e}$

$$
\Psi=\frac{10^{6} M e\left\{T_{\mathrm{E}}\right\}}{R T_{\mathrm{E}}}
$$

$\left(\mathrm{g} \cdot \mathrm{m}^{-3}\right)$ é a umidade absoluta do ar expirado; $\mathrm{M}$ é o peso molecular da água $\left(18,016 \mathrm{~g} \cdot \mathrm{mol}^{-1}\right)$, R é a constante gasosa universal $\left(8,3143 \mathrm{~J} \cdot \mathrm{mol}^{-1} \cdot \mathrm{K}^{-1}\right) \mathrm{e}$ es $\{\mathrm{TE}\}$ é a pressão de saturação de vapor do ar expirado à temperatura TE (K). Esta temperatura foi estimada como função da temperatura e da umidade do ar ambiente e da temperatura corporal dos animais, sendo usada a equação de Silva et al. (2002):

$$
\begin{array}{r}
T_{\mathrm{E}}=-142,6193+0,2891 T_{\mathrm{A}}+2,5865 e\left\{T_{\mathrm{A}}\right\}+7,3525 T_{\mathrm{B}}-0,0016 T_{\mathrm{A}}^{2}- \\
-0,2027 e^{2}\left\{T_{\mathrm{A}}\right\}-0,0797 T_{\mathrm{B}}^{2}
\end{array}
$$

$\left({ }^{\circ} \mathrm{C}\right)$, com elevado coeficiente de determinação $\left(\mathrm{R}^{2}=0,964\right)$, em que e $\{\mathrm{TA}\}$ é a pressão parcial de vapor da atmosfera à temperatura $\mathrm{TA}\left({ }^{\circ} \mathrm{C}\right)$ e $\mathrm{TB}$, a temperatura retal $\left({ }^{\circ} \mathrm{C}\right)$.

A taxa de evaporação cutânea (EC) foi estimada como a diferença entre ER e a evaporação total. Os dados foram analisados pelo método dos mínimos quadrados para dados não balanceados, usando-se o modelo:

$$
\mathrm{y}_{\mathrm{ijk}}=\mu+\mathrm{s}_{\mathrm{i}}+\mathrm{a}_{\mathrm{ij}}+\mathrm{e}_{\mathrm{k}}+\varepsilon_{\mathrm{ijk}}
$$

em que: $\mathrm{s}_{\mathrm{i}}$ é o efeito do i-ésimo sexo (i=1,2), aij é o efeito do j-ésimo animal do i-ésimo sexo, ek é o efeito da k-ésima classe de espessura do velo $(\mathrm{k}=1, \ldots 3)$, $\mathrm{m}$ é a média geral e $\mathrm{e}_{\mathrm{ijk}}$ é o efeito residual inerente à observação $\mathrm{y}_{\mathrm{ijk}}$. Partindo desse modelo, foram construídas equações lineares, as quais foram então solucionadas por um programa baseado no algoritmo iterativo de Gauss-Seidel. As somas de quadrados para os diferentes efeitos foram obtidas conforme Searle et al. (1992).

\section{Resultados e Discussão}

Na Tabela 1, encontram-se os resultados da análise de variância das taxas de evaporação respiratória e cutânea e na Tabela 2, as respectivas médias. Deve ser destacado que a expressão de ER e 
Tabela 1 - Quadrados médios das taxas de evaporação respiratória $\left(E_{R}\right)$ e cutânea $\left(E_{C}\right)$ de ovinos da raça Corriedale Table 1 - Mean squares of the respiratory $\left(R_{R}\right)$ and cutaneous $\left(E_{C}\right)$ evaporation rates in Corriedale sheep

\begin{tabular}{lccc}
\hline Fonte de variação & $\mathrm{g} .1$. & $\mathrm{E}_{\mathrm{R}}$ & \\
Source of variation & d.f. & 1 & $0,882491 * *$ \\
\hline Sexo(Sex) & 8 & $0,023313 * *$ & $1,636310^{* *}$ \\
Animais dentro sexo (Animals within sex) & 2 & $0,443544 * *$ & $0,032250 \mathrm{~ns}$ \\
Espessura do velo (Fleece thickness) & 69 & 0,007153 & $2,010138 * *$ \\
Resíduo (Residual) & & & 0,283131 \\
\hline${ }^{* *} \mathrm{P}<0,01$. & &
\end{tabular}

Tabela 2 - Médias por quadrados mínimos e erros padrões das taxas de evaporação respiratória $\left(E_{R}\right)$ e cutânea $\left(E_{C}\right)$ de ovinos da raça Corriedale $\left(\mathrm{g} \cdot \mathrm{h}^{-1} \cdot \mathrm{kg}^{-1}\right)$

Table 2 - Least squares means and standard errors of the respiratory $\left(E_{R}\right)$ and cutaneous $\left(E_{C}\right)$ evaporation rates of Corriedale sheep. Values in $\mathrm{g} \cdot \mathrm{h}^{-1} \cdot \mathrm{kg}^{-1}$

\begin{tabular}{lccc}
\hline Médias (Means) & $\mathrm{E}_{\mathrm{C}}$ & $\mathrm{n}$ & $\mathrm{E}_{\mathrm{R}}$ \\
\hline Geral (Overall mean) & 81 & $0,7599 \pm 0,0094$ & $1,3029 \pm 0,0591$ \\
Sexo(Sex): & & $0,6942 \pm 0,0145$ & $1,3924 \pm 0,0915$ \\
Machos (Males) & 58 & $0,9257 \pm 0,0199$ & $1,0772 \pm 0,1257$ \\
Fêmeas (Females) & 23 & & \\
Espessura do velo: & & & $0,8869 \pm 0,1386$ \\
Fleece thickness & 18 & $0,9389 \pm 0,0220$ & $1,4318 \pm 0,0989$ \\
$\quad 7,5-8,1 \mathrm{~cm}$ & 45 & $0,7393 \pm 0,0157$ & $1,3965 \pm 0,1386$ \\
$\quad 1,7-10,4 \mathrm{~cm}$ & 18 & $0,6326 \pm 0,0220$ & \\
$11,1-12,6 \mathrm{~cm}$ & & &
\end{tabular}

EC em termos de gramas de vapor por unidade de peso corporal é considerada como a mais correta e racional pela maioria dos autores, desde Brockway et al. (1965).

As médias gerais obtidas no presente trabalho para ER $\left(0,7599 \pm 0,0094\right.$ g.h $\left.\mathrm{h}^{-1} \cdot \mathrm{kg}^{-1}\right)$ e para EC $\left(1,3029 \pm 0,0591 \mathrm{~g} \cdot \mathrm{h}^{-1} \cdot \mathrm{kg}^{-1}\right)$ estão muito próximas das estimadas por Hofmeyr et al. (1969 em condições similares e, tal como mencionaram estes autores, a evaporação respiratória representa cerca de $37 \%$ da evaporação total. O valor de $0,702 \mathrm{~g} \cdot \mathrm{h}^{-1} \cdot \mathrm{kg}^{-1}$ observado por Hammarlund et al. (1986) dificilmente pode ser comparado com esses resultados, uma vez que foi obtido de animais recém-nascidos submetidos a temperaturas relativamente baixas $\left(22\right.$ a $\left.23,7^{\circ} \mathrm{C}\right)$.

A análise de variância mostra que houve diferença significativa entre sexos quanto a ambas as vias de evaporação: no caso da evaporação respiratória, as fêmeas apresentaram taxa média mais elevada $\left(0,9257 \pm 0,0199 \mathrm{~g} \cdot \mathrm{h}^{-1} \cdot \mathrm{kg}^{-1}\right)$ que os machos $\left(0,6942 \pm 0,0145 \mathrm{~g} \cdot \mathrm{h}^{-1} \cdot \mathrm{kg}^{-1}\right)$, enquanto no caso da evaporação cutânea os machos é que apresentaram média mais elevada $\left(1,3924 \pm 0,0915 \mathrm{~g} \cdot \mathrm{h}^{-1} \cdot \mathrm{kg}^{-1}\right.$ contra $1,0772 \pm 0,1257 \mathrm{~g} \cdot \mathrm{h}^{-1} \cdot \mathrm{kg}^{-1}$ para as fêmeas).
Hofmeyr et al. (1969) observaram para ovinos das raças Targhee, Western e Blackface expostos por duas horas a $38^{\circ} \mathrm{C}$ valores médios próximos aos obtidos no presente trabalho: $1,67 \mathrm{e} 0,90 \mathrm{~g} \cdot \mathrm{h}^{-1} \cdot \mathrm{kg}^{-1}$ nos machos e 0,92 e $0,77 \mathrm{~g} \cdot \mathrm{h}^{-1} \cdot \mathrm{kg}^{-1}$ nas fêmeas, para ER e EC respectivamente. Brockway et al. (1965) avaliaram as perdas térmicas por evaporação em animais Cheviot e seus resultados, convertidos para as unidades aqui utilizadas, indicam valores de evaporação respiratória entre $0,427 \mathrm{~g} \cdot \mathrm{h}^{-1} \cdot \mathrm{kg}^{-1}$ a $25^{\circ} \mathrm{Ce} 1,249 \mathrm{~g} \cdot \mathrm{h}^{-1} \cdot \mathrm{kg}^{-1}$ a $30^{\circ} \mathrm{C}$; quanto à evaporação cutânea, os valores foram $0,214 \mathrm{e}$ 0,286 g.h. $\mathrm{h}^{-1} \cdot \mathrm{kg}^{-1}$ a $25^{\circ} \mathrm{C}$ e $0,345 \mathrm{~g} \cdot \mathrm{h}^{-1} \cdot \mathrm{kg}^{-1}$ a $30^{\circ} \mathrm{C}$.

No que se refere à influência da espessura do velo, é interessante notar que, à medida que aumenta a mesma, reduz-se a evaporação respiratória, como é mostrado na Figura 1. O efeito da temperatura do ar sobre os dois tipos de evaporação também é diferente, conforme a espessura do velo (Figuras 2 a 4), sendo mais acentuado quando o velo é menos espesso $(7,5$ a $8,1 \mathrm{~cm})$. A evaporação cutânea tende a ser maior, à medida que a espessura do velo aumenta, até esta ser de cerca de $10 \mathrm{~cm}$; este incremento na evaporação ocorre ao nível da superfície cutânea e 
foi observado também por Hofmeyr et al. (1969), em circunstâncias semelhantes.

Uma hipótese que pode ser aventada é que o nível de evaporação cutânea, o qual se acha diretamente associado à taxa de sudação dos animais, tende a aumentar quando a temperatura da superfície da

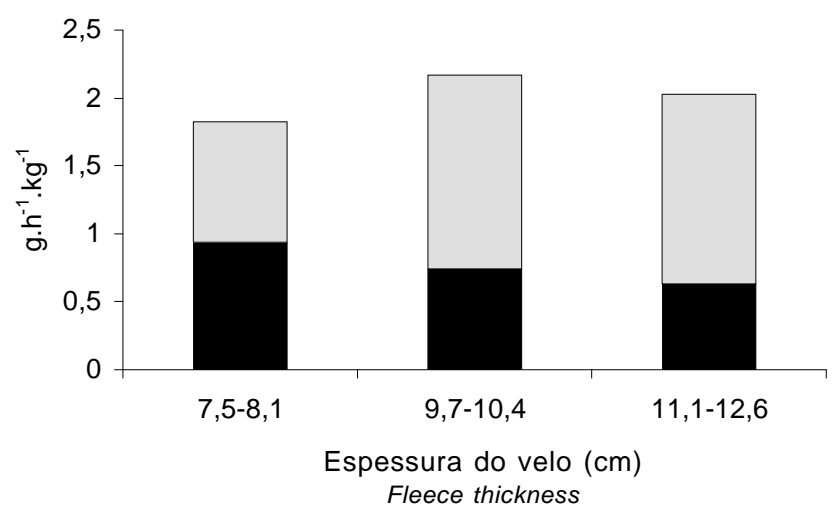

Respiratória (respiratory) $\square$ Cutânea (cutaneous)

Figura 1 - Taxas médias de evaporação respiratória e cutânea em ovinos da raça Corriedale, conforme a espessura do velo.

Figure 1 - Average respiratory and cutaneous evaporation rates of Corriedale sheep, according to the fleece thickness.

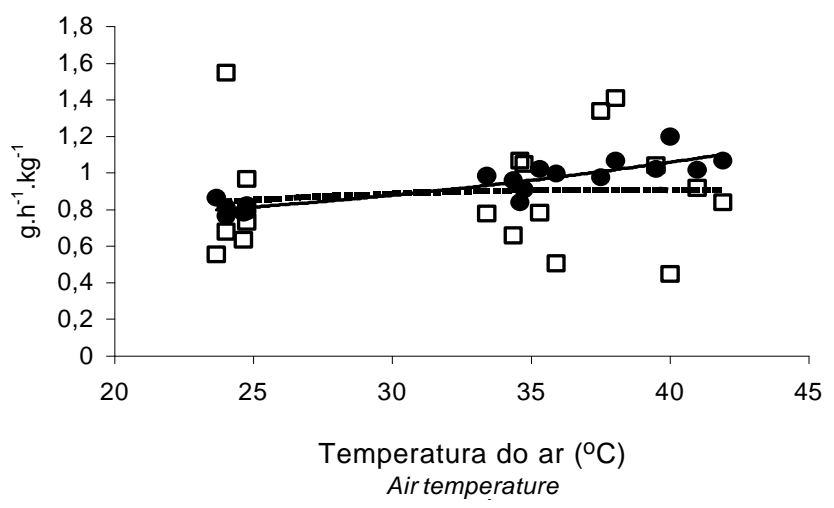

- Respiratória (respiratory)

口 Cutânea (cutaneous)

- Polinômio (Respiratória (respiratory))

- - - Polinômio (Cutânea (cutaneous))

Figura 2 - Influência da temperatura ambiente sobre as taxas de evaporação respiratória e cutânea de ovinos Corriedale com velo de 7,5 a $8,1 \mathrm{~cm}$ de espessura.

Figure 2 - Influence of the ambient temperature on the respiratory and cutaneous evaporation rates of Corriedale sheep with fleece 7.5-8.1 cm thick. epiderme se eleva. Como se pode observar na Figura 2, nos animais com velo menos espesso a evaporação cutânea permanece praticamente inalterada, à medida que a temperatura ambiente se eleva até $45^{\circ} \mathrm{C}$, ao mesmo tempo em que a evaporação respiratória sobe mais rápido nas temperaturas mais altas.

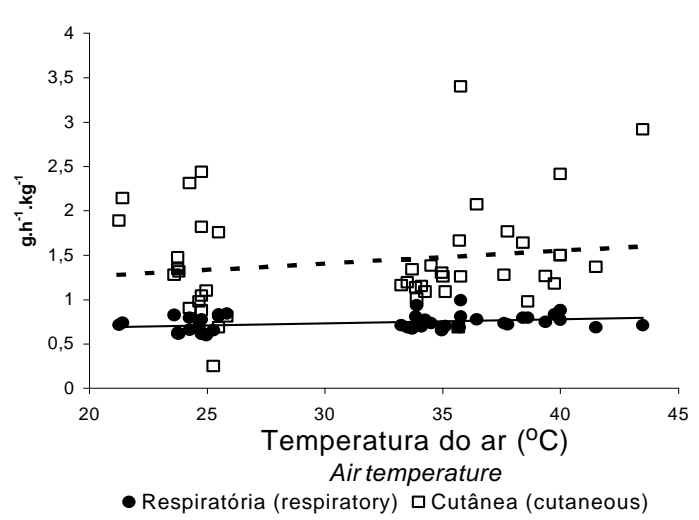

Figura 3 - Influência da temperatura ambiente sobre as taxas de evaporação respiratória e cutânea de ovinos Corriedale com velo de 9,7 a 10,4 $\mathrm{cm}$ de espessura.

Figure 3 - Influence of the ambient temperature on the respiratory and cutaneous evaporation rates of Corriedale sheep with fleece 9.7 to $10.4 \mathrm{~cm}$ thiick.

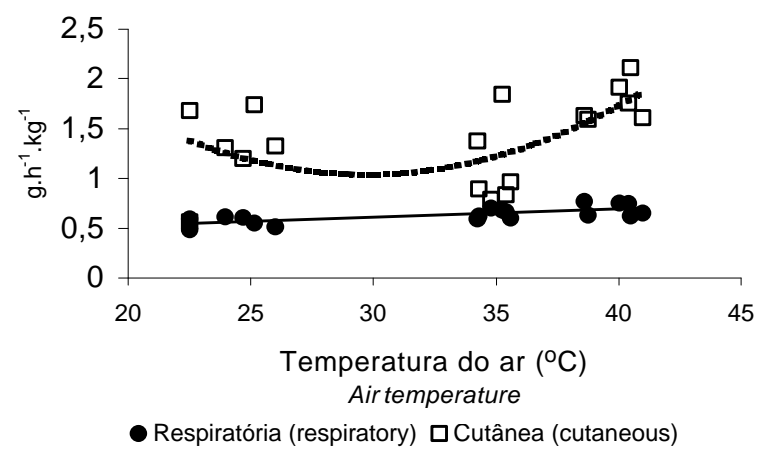

Figura 4 - Influência da temperatura ambiente sobre as taxas de evaporação respiratória e cutânea de ovinos Corriedale com velo de 11,1 a 12,6 $\mathrm{cm}$ de espessura.

Figure 4 - Influence of the ambient temperature on the respiratory and cutaneous evaporation rates of Corriedale sheep with fleece 11.1 to $12.6 \mathrm{~cm}$ thick. 
Já nos animais com velo mais espesso, a temperatura da epiderme se eleva devido à maior dificuldade de eliminação de calor sensível através da capa. Em conseqüência, a taxa de sudação aumenta e da mesma forma a evaporação cutânea. Aumentando a termólise por evaporação na superfície do corpo, o organismo pode reduzir então o trabalho respiratório, o que por sua vez resulta na diminuição da evaporação respiratória. Na Figura 5, pode-se verificar que a frequiência respiratória aumenta com o estresse térmico, indicado pela elevação na temperatura corporal. É visível uma estabilização respiratória após a temperatura corporal atingir $40,5^{\circ} \mathrm{C}$. Do ponto de vista fisiológico, isso é importante, uma vez que a frequiência respiratória muito elevada e por tempo prolongado pode causar redução na pressão sanguínea de $\mathrm{CO}_{2}$, além de sensível acréscimo no calor armazenado nos tecidos, devido ao trabalho acelerado dos músculos respiratórios.

Um aspecto que deve ser destacado é que a sudação tem sido estudada há bastante tempo em ovinos em ambientes quentes. Com respeito às raças de origem européia, têm sido observadas taxas de sudação bastante variáveis: 35 a 265 g.m $\mathrm{m}^{-2} \mathrm{~h}^{-1} \mathrm{em}$ Suffolk (Nogueira et al., 1987), 90,6 $\pm 5,5 \mathrm{~g} \cdot \mathrm{m}^{-2} \mathrm{~h}^{-1}$ em Ideal (Silva et al., 1990), 145,2 $\pm 6,1$ a $270,7 \pm 45,1 \mathrm{~g} \cdot \mathrm{m}^{-2} \mathrm{~h}^{-1} \mathrm{em}$ Rambouillet (Rai et al. 1979). Entretanto, nos ovinos lanados a epiderme é muito permeável à água e ao vapor, sendo quantitativamente muito importante a perspiração insensível, de modo

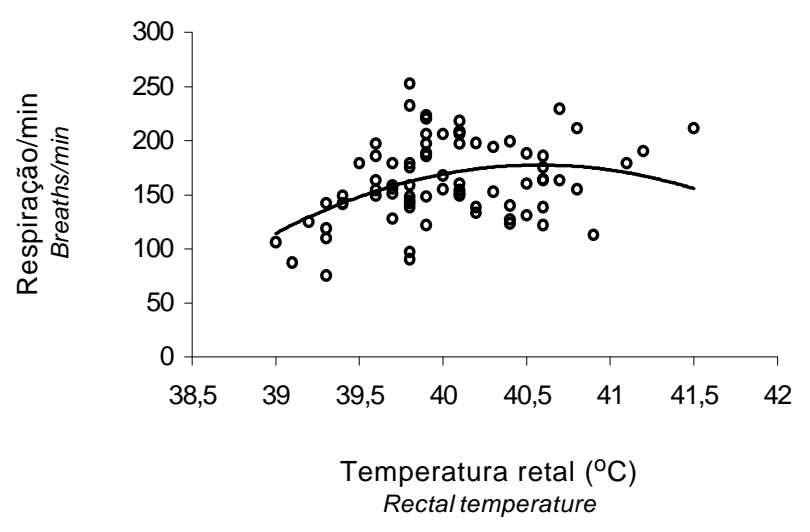

Figura 5 - Variação da frequência respiratória em função da temperatura retal, em ovinos da raça Corriedale expostos a temperaturas entre 20 e $45^{\circ} \mathrm{C}$.

Figure 5 - Variation of the respiratory rate as function of the body temperature for Corriedale sheep under environmental temperatures from 20 to $45^{\circ} \mathrm{C}$. que as taxas de evaporação cutânea geralmente correspondem a um volume de água maior que o secretado pelas glândulas sudoríparas isoladamente. Os métodos empregados na determinação da taxa de sudação não permitem a separação desta e da perspiração insensível.

De qualquer forma, os resultados obtidos no presente trabalho destacam a importância da evaporação cutânea para ovinos em ambientes quentes, confirmando as observações de Rieck et al. (1950), Knapp \& Robinson (1954) e, em parte, Hofmeyr et al. (1969). A evaporação respiratória é neste caso um mecanismo funcionalmente adequado para respostas intensas durante períodos mais curtos.

\section{Conclusões}

A taxa de evaporação respiratória (ER) para ovinos Corriedale em condições tropicais, com temperaturas ambientes entre 21 e $42^{\circ} \mathrm{C}$, é em média $0,7599 \pm 0,0094 \mathrm{~g} \cdot \mathrm{h}^{-1}$ por $\mathrm{kg}$ de peso corporal, ao passo que a evaporação cutânea (EC) é $1,3029 \pm 0,0591 \mathrm{~g} \cdot \mathrm{h}^{-1} \cdot \mathrm{kg}^{-1}$.

Há diferenças significativas entre indivíduos quanto a ER, mas não quanto a EC.

Os machos tendem a apresentar menores valores de ER e maiores valores de EC que as fêmeas.

A evaporação cutânea tem um papel importante para a termorregulação de ovinos lanígeros em ambientes de alta temperatura, contribuindo em média com $63 \%$ da evaporação total.

\section{Agradecimento}

O presente projeto foi realizado com apoio da Fundação de Amparo à Pesquisa do Estado de São Paulo (FAPESP), Proc. 97/09549-0.

\section{Literatura Citada}

ALEXANDER, G.; WILLIAMS, D. Temperature regulation in the newborn lamb. VI. Heat exchanges in lambs in a hot environment. Australian Journal of Agricultural Research, v.13, p.122-143, 1962.

BLIGH, J. The synchronous discharge of apocrine sweat glands of the Welsh mountain sheep. Nature, v.189, p.582-583, 1961.

BROCKWAY, J.M.; McDONALD, J.D.; PULLAR, J.D. Evaporative heat-loss mechanisms in sheep. Journal of Physiology, v.179, p.554-568, 1965.

BROOK, A.H.; SHORT, B.F. Regulation of body temperature of sheep in a hot environment. Australian Journal of Agricultural Research, v.11, p402-407, 1960a. 
BROOK, A.H.; SHORT, B.F. Sweating in sheep. Australian Journal of Agricultural Research, v.11, p.557-569, 1960b.

FINCH, V.A. Comparison of non-evaporative heat transfer in different cattle breeds. Australian Journal of Agricultural Research, v.36, p.497-506, 1985.

HALES, J.R.S. Physiological responses to heat. In: ROBERTSHAW, D. (Ed.) Environmental physiology. London: Butterworths, 1974.

HAMMARLUND，K.; RIESENFELD，T.; SEDIN，G. Measurement of respiratory water loss in newborn lambs. Acta Physiologica Scandinavica, v.127, p.61-65, 1986.

HOFMEYR, H.S.; GUIDRY, A.J.; WALTZ, F.A. Effects of temperature and wool length on surface and respiratory evaporative losses of sheep. Journal of Applied Physiology, v.26, p.517-523, 1969.

HOLMES, C.R. Application of a weighing system for measuring total evaporative water losses in large ruminants. Journal of Thermal Biology, v.10, p.5-7, 1985.

KNAPP, B.J.; ROBINSON, K.W. The role of water for heat dissipation by a Jersey cow and a Corriedale ewe. Australian Journal of Agricultural Research, v.5, p.568-577, 1954.

MONTEITH, J.L.; UNSWORTH, M.H. Principles of Environmental physics. 2.ed. London: E. Arnold, 1990.291p.

NOGUEIRA, O.R.; FARES, M.A.; MORÃO, F. et al. Sudação e temperatura retal em ovinos no Estado de São Paulo. In: CONGRESSO BRASILEIRO DE INICIAÇÃO CIENTÍFICA EM CIÊNCIAS AGRÁRIAS, 7., 1987, Areias. Anais... Jaboticabal: Unversidade Estadual Paulista, 1987.

RAI, A.K.; SINGH, M.; MORE, T. Cutaneous water loss and respiration rates of various breeds of sheep at high ambient temperatures. Tropical Animal Health and Production, v.11, p.51-56, 1979.
RIEK, P.F.; HARDY, M.H.; LEE, D.H.K. et al. The effect of the dietary plane upon the reactions of two breeds of sheep during short exposures to hot environments. Australian Journal of Agricultural Research, v.1, p.217-230, 1950.

ROBERTSHAW, D. Sweat gland functioning of the domestic ruminants. In: INTERNATIONAL CONGRESS OF BIOMETEOROLOGY, 4., 1966, New Brunswick, NJ. Proceedings... New Brunswick: International Society of Bometeorology, 1966.

SEARLE, S.R.; CASELLA, G.; McCULLOCH, C.E. Variance Components. New York: J. Wiley, 1992. 501p.

SILVA, R.G.; COSTA, M.J.R.P.; NOGUEIRA, O.R. Sweating rate of heat stressed sheep. In: INTERNATIONAL CONGRESS OF BIOMETEOROLOGY, 12., 1990, Viena. Proceedings... Viena: International Society of Biometeorology, 1990. p.64.

SILVA, R.G.; La SCALA Jr., N.; LIMA FILHO, A.E. et al. Respiratory heat loss in the sheep: a comprehensive model. International Journal of Biometeorology, v.46, p.136-140, 2002.

Recebido em: 15/04/02

Aceito em: 29/04/03 